\title{
Local Production, Local Consumption Peer-to-Peer Architecture for a Dependable and Sustainable Social Infrastructure
}

\author{
Kenji Saito* \\ Keio University
}

\author{
Eiichi Morino \\ Gesell Research Society Japan \\ Jun Murai \\ Keio University \\ Yoshihiko Suko
Keio University \\ Yoshihiko Suko
Keio University
}

Takaaki Suzuki

Articulate, Inc.

\begin{abstract}
Peer-to-peer $(P 2 P)$ is a system of overlay networks such that participants can potentially take symmetrical roles.

This translates itself into a design based on the philosophy of Local Production, Local Consumption (LPLC), originally an agricultural concept to promote sustainable local economy. This philosophy helps enhancing survivability of a society by providing a dependable economic infrastructure and promoting the power of individuals.

This paper attempts to put existing works of $P 2 P$ designs into the perspective of the five-layer architecture model to realize $L P L C$, and proposes future research directions toward integration of $P 2 P$ studies for actualization of a dependable and sustainable social infrastructure.
\end{abstract}

\section{Introduction}

Peer-to-peer (P2P) has potential to be a basis of a dependable and sustainable social infrastructure for enhanced survivability, because of its robustness and plasticity.

Designs of $\mathrm{P} 2 \mathrm{P}$ systems are characterized by their usage of overlay networks such that participants can potentially take symmetrical roles. This implies distribution of authorities, not only preventing introduction of single points of failure, but also possibly assuring the level of autonomy for self-organization, where any subsystem can spontaneously start, maintain itself, or recover from its failures.

This paper illustrates this potential of $\mathrm{P} 2 \mathrm{P}$ becoming a basis of a dependable and sustainable social infrastructure, and sets forth research agendas for its actualization.

\subsection{Example: power outage in Tokyo}

In August 14, 2006, there was a huge power outage in Tokyo area, affecting about 1.39 million households and

\footnotetext{
*E-mail: ks91@sfc.wide.ad.jp
}

major public transportation systems in City of Tokyo and Kanagawa prefecture (west of Tokyo) for about three hours. What caused this trouble was a crane ship, which accidentally damaged three high voltage power lines running 16 meters above water, across the river that separates Tokyo and Chiba prefecture (east of Tokyo).

The power lines convey $275 \mathrm{kV}$ of electricity generated by large power plants in Chiba to Tokyo and Kanagawa. There are two sets of such lines which can work without the other for a short period of time, but both the primary and secondary sets are supported by the same pylons. Unfortunately, the crane ship damaged one or two lines from each set, disabling them to convey enough voltage.

This incident has shown that City of Tokyo and the surrounding area had a single point of failure. Although Tokyo Electric Power Company could successfully switch the power supply to alternative routes after three hours, assuming that those alternative routes are all configured in a similar manner, it should be possible to cut off the power supply to the entire Tokyo area by just blowing up several pylons, affecting lives of more than 10 million people.

This is a kind of problem where the concept of $\mathrm{P} 2 \mathrm{P}$ becomes particularly useful.

\subsection{Philosophy: local production, local consumption}

Let us apply the concept of symmetrical roles among participants to the situation of the power supply. Then every household should be generating power, or at least every small region should be equipped with some minor power plants (perhaps using natural energy sources such as wind). In order to stabilize power supply to each household or small region, those power generators should form some network (a power grid) so that excessive power can be shared, but primarily among neighbors to prevent loss of power from long-distance conveyance and to localize the effects of accidents or sabotages. 


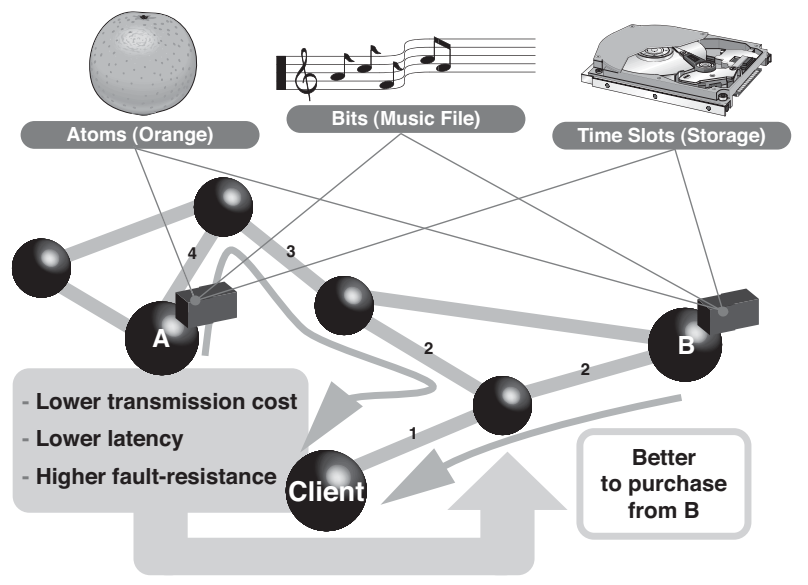

Figure 1. Purchasing goods or services on a network

This solution is generalized as follows: what consumed locally should be produced locally, and when such resources are unavailable, they should be conveyed from the nearest producers. This is the philosophy of Local Production, Local Consumption (LPLC).

LPLC is originally an agricultural term to promote sustainable local economy, which can enhance mutual understanding between producers and consumers (because they live near to each other), and has positive effects on local industry because the money spent by the local consumers do not exit from the region. But it has a wide range of applications in the designs of economic systems.

In a technological context of P2P, LPLC takes the form of emphasizing locality of communication as some systems such as Plaxton Mesh[10] or Tapestry[15] address.

As a total architecture not only for efficiency of communication, LPLC necessarily shows characteristics of P2P, because it promotes autonomy of subsystems in every scale. On the other hand, it is possible to design a $\mathrm{P} 2 \mathrm{P}$ system not to show characteristics of LPLC, as it can be designed without considering the locality of communication. Therefore, LPLC is a strengthened concept of P2P.

\section{Rationales for LPLC P2P}

The concept of LPLC has quite an engineering value, and it makes more sense to design LPLC systems than just P2P. The principle is to use the nearest resource among multiple candidates if they are considered the same. Resources include atoms (physical goods), bits (information) and time slots (labors or CPU time) as illustrated in Figure 1.

\subsection{Purchasing atoms}

Let us consider the case of purchasing oranges online.

We cannot evaluate the values of the oranges easily because we cannot take them on our hands or taste them. Trust with the venders or producers (for the sake of arguments, they are called producers altogether henceforth) is also a problem; it is possible that the producers do not send the goods to us after receiving money for them.

Perhaps if there are predecessors who have made transactions with the producers before, they could provide useful information. A reputation system will be useful.

Suppose we could successfully evaluate the qualities of the oranges. If there are several producers with the same grade of oranges, it makes more sense if we purchase them from the one nearest to us in the logistic network. Then the oranges will be fresher, and possibly cheaper because of smaller transportation cost. Moreover, if a road is closed somewhere because of some disaster, it is more probable that we can still purchase oranges from the nearest producer. LPLC is a rational strategy in an economic sense.

Note that this is always a question of a distance within a (logistic) network, which does not necessarily equal the physical distance.

\subsection{Purchasing time slots}

Let us then consider purchasing some storage service from another computer on a P2P overlay network. As it is the case for purchasing oranges, the quality of the services cannot be predetermined (availability of peers, performance of disks, etc.). Trust is also a problem because the peers might not give access to the storage after receiving the fee.

Perhaps if there are predecessors who have made transactions with the peers before, they could provide useful information. Again, a reputation system will be useful.

Suppose we could determine the quality of the storage services. Then it makes more sense if we purchase the service from the computer nearest in the communication path among the ones who provide the same level of services, as it will have less delay, less affected by faults because it passes through smaller number of failure points, and if the network is divided, more certain to be able to use the service.

Again, LPLC is more economical, and provides more survivability.

\subsection{Purchasing bits}

Purchasing bits is another story because bits can be copied exactly at low cost, making the concept of producers ambiguous.

However, if there are the same music files on different paths from a consumer, for example, it will make more 


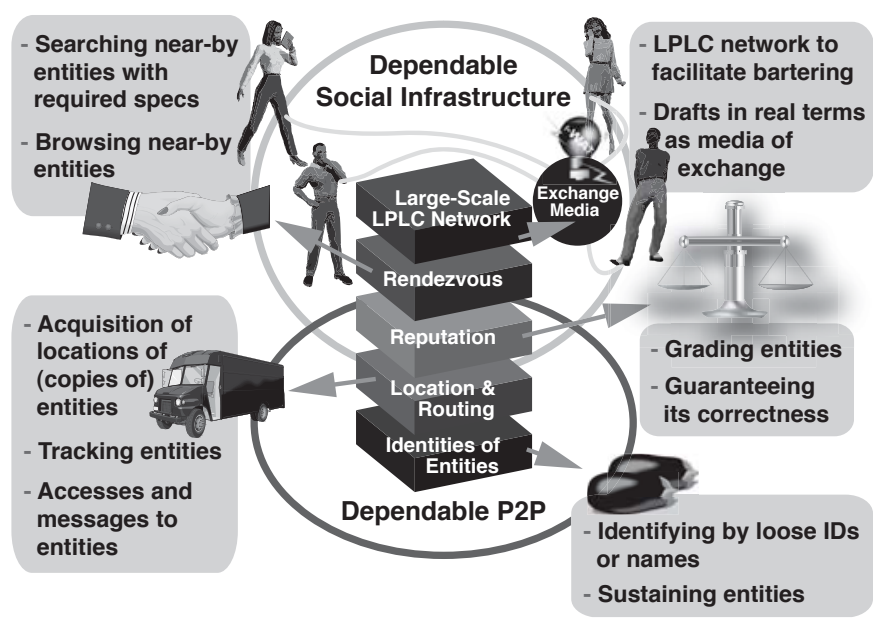

Figure 2. Five-layer architecture model

Table 1. Five-layers and existing works

\begin{tabular}{r||l}
\hline \multicolumn{1}{c||}{ Layers } & \multicolumn{1}{c}{ Existing research works } \\
\hline Large-scale LPLC network & $\begin{array}{l}\text { BitTorrent[3], Samsara[4], MojoNation[1], } \\
\text { Karma[13], Ripple[7], PPay[14], } i \text {-WAT[11] }\end{array}$ \\
\hline Rendezvous & Peer Group Rendezvous[5] \\
\hline Reputation & EigenTrust[8], Stamp trading[9] \\
\hline Location and routing & Chord[12], Tapestry[15], etc. \\
\hline Identities of entities & Freenet[2], Ecosystem of Naming Systems[6] \\
\hline
\end{tabular}

sense to access the nearest copy for the reasons we have just investigated. Some lookup systems, such as Tapestry, are designed to exploit this type of economy.

\section{$2.4 \quad$ LPLC as a fundamental}

LPLC is also a fundamental concept underlying many of the hot topics of networking: Ubiquitous computing is about amenities being always provided by near-by entities. Location-based information system is about selecting entities by vicinity to provide information. Sensor network makes sensor information particularly useful for near-by entities. Mobility means that a sustained image of services is provided by a group of near-by entities even when the consumers move. Ad-hoc networking creates a field of information with near-by entities.

\section{Five-layer architecture model for LPLC}

The question now is how we design LPLC P2P systems. Another question is how close the $\mathrm{P} 2 \mathrm{P}$ research community is to actualization of LPLC.

The authors have made the five-layer architecture model (Figure 2), into which we have attempted to put existing works of P2P designs as shown in Table 1.

\subsection{The perspective}

The model is designed in a top-down way from the uppermost layer of large-scale LPLC network.

Some economic system is necessary to make a system usable in reality if we share time slots such as storage or bandwidth over a P2P overlay network. For example, BitTorrent[3] has a mechanism of choking the download bandwidth to peers who do not provide upload bandwidth.

In order for those facilities for purchasing or exchanging goods or services to work, one needs to determine what to purchase or exchange, by searching near-by entities satisfying required specifications (rendezvous). For such searches to work, grading entities in a distributed manner and guaranteeing its correctness are required (reputation).

For the reputation systems to work, as well as other basic communications and conveyance, we need to locate entities and forward messages to them (location and routing). To do so, we need the most fundamental mechanisms of identifying entities, assuring anonymity of them if necessary (identities of entities).

\subsection{Future research directions}

Currently, the focuses of the $\mathrm{P} 2 \mathrm{P}$ research community are mostly on location and routing and identities of entities, into which many existing works fall.

The authors believe that we need to shift our focuses to the upper layers, and at the same time, redesign the lower layers based on the requirements from the upper layers.

To proceed, we need to determine how the uppermost layer is designed.

\section{Designs of the exchange media}

An LPLC network requires exchange media, otherwise it always has to require an unrealistic condition of double coincidence of wants ${ }^{1}$. The question we need to ask ourselves is how we design digital exchange media while bits can be easily copied (even if they are protected, people will find a way). An answer may be that some bits will not be copied easily (people will not want to), such as bits as a proof of debt of the holder.

\subsection{Commodity money in LPLC network}

Autonomous economy, where participants can generate exchange media themselves, is not a new concept. In old Japan, rice was a common value being used as a medium

\footnotetext{
${ }^{1}$ A situation in which $A$ wants what $B$ can provide, and $B$ wants what $A$ can provide, as it is the case for the mechanism of BitTorrent. This is still useful in solving specific economic problems in $\mathrm{P} 2 \mathrm{P}$.
} 
of exchange (commodity money). In P2P overlay networks, such common values are rather found easily.

Samsara[4] is a fair P2P storage infrastructure in which each peer that requests storage of another must agree to hold a claim, or incompressible space, in proportion to their consumption. Claims can be forwarded along the chain of nodes (that requests storage of another), eliminating themselves when cycles are found.

The authors would argue that claims can be forwarded in exchange with services other than storage, making them commodity money in the context of P2P (services and claims go the same direction). This is a possibility we would like to pursue, although some notable problems, particularly the efficiency of storage and bandwidth, may arise.

\subsection{Drafts in real terms}

If rice is a common value, promise of a specific amount of rice of a specific grade can become a guarantee believable enough to work as money. This is the concept of drafts in real terms, which was a exchange medium used in Japan in around 12 th-13th century. If we are to receive rice of the same grade in exchange of such a medium, the nearer the producer is, the cheaper the transactional cost is. Therefore this medium connotes the concept of LPLC.

$i$-WAT[11] is a protocol which can implement such drafts in real terms in an electronic way. It is a digital version of the WAT System, a barter currency, whose medium is a ticket resembling a bill of exchange.

By issuing a digital ticket promising a certain grade of storage service, a peer in a P2P overlay network can purchase a service from another. Those tickets can be forwarded along the chain of nodes in exchange with services (services and tickets go opposite directions), eliminating themselves when they return to their issuers. This is another possibility we would like to pursue; actually, we have been experimenting with usages of $i$-WAT for several years.

\section{Conclusions}

LPLC, a strengthened concept of P2P, is a good philosophy for designing sustainable, cooperative (with nature), efficient, dependable social infrastructure that can support our lives.

We have made the five-layer architecture model to realize LPLC, and attempted to put existing research works in the areas of $\mathrm{P} 2 \mathrm{P}$ so that we can set forth research agendas towards actualization of LPLC.

We introduced two candidates for the designs of exchange media in LPLC networks that can coexist: claims for storage, originally an idea from Samsara, and $i$-WAT tickets as drafts in real terms. We will investigate those possibilities further in the future.

\section{References}

[1] AZI. Mojo Nation technology overview. Online archive. Available electronically at http://web.archive.org/web/20020127125928/

www.mojonation.net/docs/technical_overview.shtml.

[2] I. Clarke, O. Sandberg, B. Wiley, and T. W. Hong. Freenet: A distributed anonymous information storage and retrieval system. In Proceedings of the ICSI Workshop on Design Issues in Anonymity and Unobservability, 2000.

[3] B. Cohen. Incentives build robustness in bittorrent. In Proceedings of the First Workshop on Economics of Peer-toPeer Systems, May 2003.

[4] L. Cox and B. Noble. Samsara: Honor among thieves in peer-to-peer storage. In Proceedings of the ACM Symposium on Operating Systems Principles, October 2003.

[5] Y. Doi. Peer group rendezvous using intersection among peer groups on DHT. In Proceedings of 2004 Symposium on Applications and the Internet (SAINT 2004 Workshops). IEEE Computer Society Press, January 2004.

[6] Y. Doi, S. Wakayama, M. Ishiyama, S. Ozaki, T. Ishihara, and Y. Uo. Ecosystem of naming systems: Discussions on a framework to induce smart space naming systems development. In Proceedings of the First International Workshop on Dependable and Sustainable Peer-to-Peer Systems (DASP2P 2006), pages 772-778, April 2006.

[7] R. Fugger. Money as IOUs in social trust networks \& a proposal for a decentralized currency network protocol. Hypertext document. Available electronically at http://ripple.sourceforge.net/.

[8] S. D. Kamvar, M. T. Schlosser, and H. Garcia-Molina. The EigenTrust algorithm for reputation management in P2P networks. In Proceedings of the Twelfth International World Wide Web Conference, 2003.

[9] T. Moreton and A. Twigg. Trading in trust, tokens, and stamps. In Proceedings of the Workshop on the Economics of Peer-to-Peer Systems, June 2003.

[10] C. G. Plaxton, R. Rajaraman, and A. W. Richa. Accessing nearby copies of replicated objects in a distributed environment. In Proceedings of ACM SPAA, June 1997.

[11] K. Saito. $i$-WAT: The Internet WAT System - An Architecture for Maintaining Trust and Facilitating Peer-to-Peer Barter Relationships - . PhD thesis, Graduate School of Media and Governance, Keio University, February 2006.

[12] I. Stoica, R. Morris, M. F. K. David Karger, and H. Balakrishnan. Chord: A scalable peer-to-peer lookup service for internet applications. In Proceedings of ACM SIGCOMM, August 2001.

[13] V. Vishnumurthy, S. Chandrakumar, and E. G. Sirer. KARMA: A secure economic framework for $\mathrm{p} 2 \mathrm{p}$ resource sharing. In Proceedings of the Workshop on the Economics of Peer-to-Peer Systems, June 2003.

[14] B. Yang and H. Garcia-Molina. PPay: micropayments for peer-to-peer systems. In Proceedings of the 10th ACM conference on Computer and communications security (CCS '03), October 2003.

[15] B. Y. Zhao, J. D. Kubiatowicz, and A. D. Joseph. Tapestry: An infrastructure for fault-tolerant wide-area location and routing. Technical Report UCB//CSD-01-1141, U.C.Berkeley, April 2001. 